\title{
Response to letter to the editor OSIN-D-20-00441 re: "Denosumab-induced hypocalcemia in patients with osteoporosis: can you know who will get low?"
}

\author{
G. Tsvetov $^{1,2}$ (D) O. Amitai ${ }^{1,3} \cdot$ T. Diker-Cohen $^{1,2,4}$ (D) \\ Received: 19 May 2020 / Accepted: 3 June 2020 / Published online: 12 June 2020 \\ (C) International Osteoporosis Foundation and National Osteoporosis Foundation 2020
}

We thank Ho et al. for their interest in our study and fully agree with their insightful comments.

As stated by Ho, there is no clear protocol for routine monitoring of calcium levels before or during treatment with denosumab. This was our incentive to assess the incidence of denosumab-associated hypocalcemia (DAH) in a large community-based cohort of patients. Most of these patients were treated in primary care clinics. This probably contributes to the major difference in the rate of hypocalcemia observed in our study versus that observed by Ho et al. In our study, the large variability in timing of calcium tests after the start of denosumab therapy suggests that these were random checks rather than tests aimed at detection of treatment-associated adverse events. Moreover, 32 of the patients with hypocalcemia at baseline received denosumab and were excluded from our study. This suggests that awareness to DAH should be increased. In the study by Ho et al., patients were closely monitored. Their results support the notion that vitamin D repletion, adequate calcium and vitamin $\mathrm{D}$ supplementation, and regular follow-up of serum calcium are the key to safe treatment with denosumab in high-risk patients.

Previous studies observed a high rate of DAH in patients with end-stage renal disease [1]. Therapeutic options are limited for these patients who have complex metabolic bone disease. We eagerly await the results by Ho et al. in patients with

T. Diker-Cohen

taliadico@gmail.com

1 Institute of Endocrinology, Diabetes and Metabolism, Rabin Medical Center - Beilinson Hospital, 39 Jabotinski St., 4941492 Petach Tikva, Israel

2 Department of Medicine A, Rabin Medical Center - Beilinson Hospital, Petach Tikva, Israel

3 Clalit Healthcare Services, Dan-Petach-Tikva district, Israel

4 Sackler Faculty of Medicine, Tel Aviv University, Tel Aviv, Israel progressive kidney dysfunction treated with vitamin D versus calcitriol.

Gloria Tsvetov, MD

And Talia Diker-Cohen, MD PhD

Department of Endocrinology, Diabetes \& Metabolism

Rabin Medical Center - Beilinson Hospital

39 Jabotinski St., 4941492, Petach Tikva, Israel

\section{Compliance with ethical standards}

Conflict of interest None.

\section{Reference}

1. Thongprayoon $\mathrm{C}$, Acharya $\mathrm{P}$, Acharya $\mathrm{C}$, Chenbhanich J, Bathini T, Boonpheng B, Sharma K, Wijarnpreecha K, Ungprasert P, Gonzalez Suarez ML, Cheungpasitporn W (2018) Hypocalcemia and bone mineral density changes following denosumab treatment in endstage renal disease patients: a meta-analysis of observational studies. Osteoporos Int 29:1737-1745. https://doi.org/10.1007/s00198-0184533-6

Publisher's note Springer Nature remains neutral with regard to jurisdictional claims in published maps and institutional affiliations. 